The Authors' accepted manuscript pre-publication; may contain typographic errors. Full citation:

McNarry, G, Allen-Collinson, J \& Evans, AB (2020) 'Doing' competitive swimming: Exploring the skilled practices of the competitive swimming lifeworld, International Review for the Sociology of Sport, online early.

https://journals.sagepub.com/doi/pdf/10.1177/1012690219894939

\title{
'Doing' Competitive Swimming: Exploring the skilled practices of the competitive swimming lifeworld
}

\author{
Gareth McNarry, PhD*/** \\ Jacquelyn Allen-Collinson, PhD* \\ Adam B Evans, PhD**
}

* Health Advancement Research Team (HART), School of Sport \& Exercise Science, University of Lincoln, UK

** Department of Nutrition, Exercise and Sports, University of Copenhagen, Denmark

Corresponding author: Dr Gareth McNarry, University of Lincoln, HART, School of Sport \& Exercise Science, Brayford Campus, Lincoln LN6 7TS, UK 


\begin{abstract}
Despite a developing literature on various facets of sporting embodiment, there is currently a research lacuna with regard to in-depth analyses of actually 'doing' sporting activities within specific physical cultures. In this article we address that gap by drawing on a developing theoretical literature in sociological phenomenology to investigate a particular physicalcultural domain. Here, we present and analyse data from an ethnographic study of competitive swimmers undertaken in the United Kingdom (UK). Responding to calls to explore the domain of 'body pedagogics', we investigate the embodied work involved in the skilled practice of 'doing' and learning how to 'do' competitive swimming. This embodied work plays a key part in the swimmers' ability to inhabit the competitive swimming lifeworld. In the analysis, we highlight how the acquisition and 'habituation' of these body techniques and skilled behaviors is not achieved simply through the repetitive rehearsal of coherent movements over time. These processes are complex, demanding practical experimentation, discovery, and the ability constantly to adapt to changes in the environment and the swimmer's own corporeality.
\end{abstract}

Keywords: sociological phenomenology; competitive swimming; body pedagogics; doing; habit

\title{
Introduction
}

Despite a developing literature that focuses on various facets of sporting embodiment, there is currently a research lacuna with regard to in-depth analyses of actually 'doing' sporting activities within specific physical cultures. In this article, we address that gap by drawing on a developing theoretical literature that employs a sociological form of phenomenology to investigate particular physical-cultural domains, and place the focus on competitive swimming. Responding to calls to explore the domain of 'body pedagogics' (Shilling, 2017), we investigate the embodied work involved in the skilled practice of 'doing' and learning how to 'do' competitive swimming, presenting data from an ethnographic study of competitive swimmers undertaken in the United Kingdom (UK). This embodied work plays a key part in the swimmers' ability effectively to inhabit the competitive swimming lifeworld. In the analysis, we highlight how the acquisition and 'habituation' of these body techniques and skilled behaviors is not achieved simply through the repetitive rehearsal of coherent 
movements over time. These processes are complex, demanding practical experimentation, discovery, and the ability constantly to adapt to changes in the environment and the swimmer's own corporeality.

Competitive swimmers regularly spend between 10 and 25 hours per week in the water, covering anywhere from 25 to over 100 kilometers of swimming. This volume of work is widely accepted within the swimming 'lifeworld' (the world of everyday life as subjectively and intersubjectively experienced) as the norm and is often adopted early in a swimmer's career, usually during adolescence. In addition to this volume of pool-based training, such swimmers will often supplement their pool-work with land-based training such as weighttraining, circuits, and 'spinning' (cycling in a gym setting). Swimming at this level requires the development of a significant level of corporeal proficiency through the acquisition and continued refinement of a variety of body techniques (Mauss, 1979). The aim of this article is to explore and describe the most prominent of these body techniques and the development of corporeal ways of knowing and 'doing' competitive swimming; a process that demands constant monitoring and (re)evaluation of skilled movements and techniques. Hence, our purpose is to investigate the nexus of mind-body-environment in sporting experience, which remains sociologically under-researched, particularly in relation to Shilling's (2017) call for sociology to address body pedagogics: an embodied approach to the acquisition and transmission of sporting, occupational, and other culturally-structured practices.

Interestingly, much of the extant sociological research on competitive swimming utilizes a Foucauldian perspective. For example, McMahon et al. (2012) liken the training practices undertaken by competitive swimmers in the Australian swimming culture, enforced on them by coaches and team managers in the name of performance, to Foucault's notion of 'discipline', leading to the creation of docile bodies. McMahon and Penney (2013a; 2013b) also draw upon Foucault's notions of 'surveillance' and 'the gaze' to highlight how within a sporting context the body can be subjected to visual scrutiny from many eyes, including those of coaches, team-mates, parents and the self. Lang (2010) also draws attention to the surveillance and conformity of athletes, highlighting how the pressure of constant surveillance leads to athletes submitting to intensive training protocols and normalized practices. Whilst these studies are helpful in illuminating the disciplinary forces at work in this particular physical culture, these studies often explore what is done to the body by various social-structural forces and power relations, rather than what is done by the body. In contrast, 
here we embrace an embodied perspective that investigates the lived, corporeally-grounded experience of competitive swimming, in order to illuminate a perspective from the competitive-swimming body, whilst acknowledging that social-structural forces strongly shape this experience.

In doing so, we draw connections with Throsby's (2016) 'aquatic sociology'. Her work highlights the body techniques that marathon swimmers incorporate and embody reflexively in order to swim long distances, and belong to the wider community of marathon swimming. We further develop Throsby's work by contributing insights from competitive poolswimming. Crossley's (1995) notion of a 'carnal sociology' is also germane to our work in addressing aquatic experiences of 'intense embodiment' (Allen-Collinson and Owton, 2015). We therefore portray the social actor not just as a wielder of symbols, but as a 'sensate, suffering, skilled, sedimented and situated creature of flesh and blood' (Wacquant, 2015: 1). Before describing the research from which our embodied data derive, we first provide a brief overview of our theoretical framework of sociological phenomenology.

\section{Sociological phenomenology and sporting embodiment}

Originated in its modern-day transcendental form by the German philosopher, Edmund Husserl (1970), phenomenology has subsequently developed and differentiated into a complex array of different traditions (Allen-Collinson, 2009), including existential and hermeneutic strands. The term itself derives from the Greek phainomenon, with the root phôs, or light; phainomenon is therefore that which is placed in the light, made apparent or shown to us. From this is derived the English phenomenon: an appearance, an observable occurrence. Phenomenology thus constitutes the study of phenomena, things as they present themselves to, and are perceived in, consciousness (Allen-Collinson, 2016). Noumena, in contrast, are considered the objects 'producing' the phenomena, although the notion of an object's independent existence remains contested within different philosophical traditions. Intentionality was a key element in Husserl's (1970) oeuvre, developed from Brentano's (1874/1995) earlier thinking, and highlighting that human consciousness is always directed toward something. The relationship between human consciousness and the world lies at the heart of phenomenology, and studying phenomena requires the phenomenologist temporarily to engage in the epochē (a form of bracketing). This process requires us to 'take a step back' from the world of everyday, and from our own taken-for-granted understandings 
- termed the 'natural attitude' - in order to contemplate what it is to be a participant in the world, and how things present themselves to us (Sokolowski, 2000). In brief, the aim is to approach the phenomenon under study with an open and questioning attitude, suspending as far as possible our taken-for-granted, everyday assumptions and presuppositions.

Whilst Husserl's focus was primarily on the mind-world nexus, existential phenomenologists such as Maurice Merleau-Ponty (2002) have highlighted the centrality of our corporeality in lived experience; the body as the point from which we perceive and 'live' the world from a first-person perspective (the perspective of 'le corps propre'/one's own body). This 'lived body' links mind-body-world in an indissoluble relationship, so that mindbody-world constitute part of the very fabric or 'flesh' of the world, as Merleau-Ponty (1968) argues in his later, and unfinished work, which moves away from the traditional phenomenological focus upon the primacy of consciousness. Of further note, and drawing from his earlier work, Merleau-Ponty (2002: xx) takes the Husserlian notion of intentionality and identifies a specific variant: operant or operative intentionality, which refers to the prereflective and corporeal link that a subject has with the world.

Moving to consider our specific form of sociological phenomenology, Husserl's phenomenology was first applied within (North American) sociology by Alfred Schütz (1967) whose sociological attention was piqued particularly by the Husserlian conception of the lifeworld (Lebenswelt). Schütz adapted and developed aspects of Husserl's thinking to create a phenomenological-sociology or sociological-phenomenology (Wagner, 1973). Here, we thus draw upon what has been conceptualised as phenomenologically-inspired sociology or 'sociologized' phenomenology (Allen-Collinson, 2009, 2011), a phenomenological form that diverges from philosophical phenomenology, to acknowledge the myriad effects of culture and social structure upon lived-body experience. Whereas philosophical phenomenology seeks to identify 'essences' of phenomena, fundamental and universal patterns or structures of experience spanning all time and all people, sociological phenomenology acknowledges the possibility of 'difference' in some aspects of lived experience. Feminist phenomenologists, for example, highlight the role of sex or gender difference in lived embodied experience (e.g. Fisher and Embree, 2000).

The empirical, sociological form of phenomenology (Allen-Collinson, 2016) we utilize thus draws upon Merleau-Ponty's (2002) existential phenomenology in highlighting the centrality of the body in human experience, together with the conceptualization of world, 
body and human consciousness as fundamentally intertwined and mutually influencing (Allen-Collinson, 2009). Given the centrality of the body in doing sport, it is perhaps surprising that this form of existential phenomenology has not been employed more widely in the study of sport (Allen-Collinson, 2009; Kerry and Armour, 2000). A growing corpus is, however, beginning to develop, for example, in relation to Mixed Martial Arts (MMA) (Vaittinen, 2014), distance running (Hockey and Allen-Collinson, 2016), dance (Purser, 2019), and rowing (Ravn and Christensen, 2014), to which we contribute our data on the lifeworld of competitive swimming.

Germane to our analysis are other theoretical perspectives that similarly draw upon corporeally-contoured insights, such as Mauss' (1979) 'techniques of the body' that portray the mental, physical and social aspects of human being as an irreducible whole. Skills and techniques thus become habit, but not as mere mechanical repetitions of action, but rather as the ability to grasp and incorporate within one's body schema a tacit and practical 'principle' of action that permits new ways of acting and understanding, creating the 'habitbody' (Crossley, 2001: 127). Leder's (1990) phenomenologically-inspired conceptualization of the 'dis-appearing' and 'dys-appearing' body is also pertinent here, as is that of Zeiler's (2010) 'eu-appearing body ${ }^{1}$. For Leder (1990), the body is generally in a state of 'dis-appearance' during the general flow of normal day-to-day activity, remaining largely absent from our consciousness. Our focus is not inward to our body but outward to the world, and our body remains in the 'background'. In contrast, the 'dys-appearing' body is characterized by a state of 'dys-ease' when the body shifts position from backgrounded presence to constitute the direct focus of consciousness, for example, when pain or illness disrupts the body-world relationship and commands attention. Moreover, the more positive and even pleasurable aspects of such body intentionality have been highlighted by Zeiler (2010: 334), who notes, 'the subject can attend to her or his body as something positive and that this attention need not result in discomfort or alienation'.

Having given a brief overview of our phenomenologically inspired approach, we now delineate the research project in which we investigated the phenomenological nexus of mindbody-environment in sporting experience. This domain remains sociologically under-

\footnotetext{
${ }^{1}$ Eu-appearance - Zeiler (2010) uses the Greek prefix eu to highlight the experience of the body as well, easy or good.
} 
researched, particularly, as highlighted above, vis-à-vis Shilling's (2017) call for sociology to investigate body pedagogics as an embodied approach to the acquisition of sporting practices - in our case within the culture of competitive swimming.

\section{The research}

Ethical approval was granted by the relevant university ethics committee prior to data collection. Ethnographic data were gathered by the first author during a full swimming season (September to July) from two performance groups of competitive swimmers comprising 36 swimmers, aged $18-22$, based at one of the top ranked sporting universities in the UK. The University has a long history of producing senior performance swimmers with numerous international representations and honours. As we have noted elsewhere (McNarry et al., 2019), phenomenology is not a method in the traditional sense of a research technique, but is an approach that embraces a whole way of thinking and reflecting, characterized by an attitude of openness, curiosity and ceaseless questioning of the taken-for-granted (AllenCollinson, 2009; 2016). Indeed, Merleau-Ponty (2002: xv) highlights that to see the world phenomenologically we must: 'break with our familiar acceptance of it', temporarily bracketing our existing pre-suppositions and assumptions via the process of epoche (see also Allen-Collinson, 2011). One of the key initial challenges, therefore, was for the primary researcher to undertake bracketing conversations with the supervisory team, who sought to 'unveil' and problematize his tacit knowledge and assumptions about the lifeworld of competitive swimming; a world with which he was very familiar through his own lived experience.

The primary researcher adopted the role of a volunteer assistant; a familiar role given that he had been both a swimmer and swimming coach, making him what some have termed a 'cultured insider' (Evers, 2006; Wheaton, 2002). This allowed him to select information-rich cases in the form of participants with direct lived experience of the performance-swimming lifeworld, and permitted him privileged access to the field site where, in total, over 300 hours of observations were completed across three, five-week immersions: 2 October to 4 November, 2017; 24 January to 28 February, 2018; 20 June to 21 July 2018. Observations were recorded via the OneNote application on the first author's iPhone, with subsequent detailed notes written up immediately following each session. These notes recorded what was going on in the session, what the swimmers did, how they did it, and how it seemed to 
affect them. Notes were taken not only about specific incidents or moments, but also regarding the normal, everyday routines of the swimming sessions. Most of the notes were written in the form of a first-person narrative, allowing for the inclusion of the primary researcher's own reflections from the field, and providing him with a space to reflect upon the more nuanced individual behaviours he observed. Data were also collected via semistructured interviews (19 individual and three group interviews), with sampling criteria for interviewees constituting: 1 ) being an active member of one of the performance swimming squads; and 2) having competed at a minimum of British Championship level in the previous 12 months. These criteria ensured that participants had a high level of commitment to, and familiarity with the sport, and thus were in a position to provide rich, in-depth descriptions of their aquatic lifeworld.

The interviews drew inspiration from Kvale and Brinkmann's (2009) 'lifeworld' interview process, which is commensurate with a phenomenologically-sensitive approach, and: 'attempts to understand themes of the lived everyday world from the subject's own perspectives' (Kvale and Brinkmann, 2009: 27). Although some sports psychologists (Dale, 1996; Nesti, 2004) have argued that a more unstructured phenomenological interview is better suited to a phenomenological study, in this case such an approach would have been problematic for a number of reasons, including the age of the participants, the challenging demands of a phenomenological interview, and the time necessary for an effective encounter to take place. In total, 19 athletes (12 men and 7 women) were interviewed, spanning a range of experience: of different swimming strokes, events, and level of competition - from British Championship level through to those who had competed internationally including at the World Championships and the Commonwealth Games.

Initial individual interviews were recorded on a Dictaphone during 'immersion one' and transcribed verbatim. A follow up interview was held with each participant during 'immersion two' to gain further information and (if needed) clarification on comments made in the initial interview. Three final group interviews were conducted during 'immersion three' as a means of engaging with the process of member reflection in order to permit sharing and dialoguing with participants about the study's findings, and providing opportunities for questions, critique, feedback, and affirmation (Tracy, 2010). This was not considered a 'memberchecking' procedure, but rather as a further data collection opportunity in which we sought to: 'yield new data which throws fresh light on the investigation and which provides a spur 
for deeper and richer analyses' (Bloor, 2001: 235). Data analysis was undertaken via the reflexive thematic analysis (TA) approach outlined by Braun and Clarke (2013; 2019). This involved the primary researcher familiarizing himself with the data by listening to and reading the various transcripts on several occasions, then generating initial codes, searching and defining themes, and discussing these in detail with research supervisors. TA is thus more an 'adventure than a recipe' (Braun and Clarke, 2019: 4) where one moves back and forth as required between the data and the analysis, refining and redeveloping the generated themes and concepts in order to construct knowledge from the data. In the data extracts that follow all participants have been given pseudonyms, and their ages are included. We have, however, not provided greater detail of participant characteristics, in order to protect their anonymity, given the relatively small overall population of British university performance swimmers and the likelihood of identifiability. Several key themes were identified in the data cohering around the ways in which competitive swimmers engage with 'doing' swimming: 1) the process of mindful swimming, to maintain efficiency and speed; 2) mindful changing and development of technique; 3) the pre-reflective, 'switched off' swimming of recovery sessions.

\section{The mindful 'doing' of competitive swimming}

A key feature to the process of 'doing' competitive swimming is understanding that 'doing' involves, as Throsby (2016: 28) notes, a 'self-orientated project of perpetual improvementfocused embodied labor that is accomplished reflexively and iteratively'. Each time swimmers enter the water (or the gym), their aim is to improve as an athlete. The competitive swimming body thus is an ongoing embodied project that is continuously worked on, in a social and cultural context where the body is deemed both malleable through hard work and vigilance, as well as being a social symbol that 'gives off' messages about the self (Shilling, 2012: 7). The competitive swimming-body, like Throsby's (2016) marathon swimming body, is therefore a body that is 'made' via hard work and commitment. The swimmers in this study, however, indicated holding views of the competitive swimming body as being both 'born' and 'made'; a combination of genetic luck and the undertaking of considerable embodied labor:

...of the people who have been given certain physical benefits, out of that group of people it's the ones that work hard out of them that will make it, do you know what I mean. So, I think you need both (Stephen, 20). 
A key element of this embodied labor is the acclimatization to a variety of swimmingspecific body techniques that characterize the competitive swimming body and give shape to the competitive swimming habitus. Here we employ a phenomenological conceptualization of the habitus, which is seen as a 'lived-through structure-in-process, constantly evolving as an effect of the interactions of the agent or group with both others and their physical environment' (Crossley, 2004: 39). This interactive process involves the development and refinement of habituated bodily actions that become part of the individual's corporeal schema. There is thus a specific mode of being, doing, and understanding that swimmers have to incorporate and embody in order to engage effectively and cope with the physical and psychological demands of the sport, as well as to belong to the wider community of competitive swimmers. These 'techniques of the body' (Mauss, 1979) or 'habits' (MerleauPonty, 2002) are acquired through a process of apprenticeship in culturally-specific contexts (Shilling, 2010) as a form of body pedagogics (Allen-Collinson et al., 2018; Shilling, 2017), and are essential to the production of the competitive swimming body and lifeworld. Such acquisition requires the ability not only to swim, but to swim effectively, efficiently, and ultimately quickly. While there may be a minimum standard below which competitive swimming becomes untenable (e.g. failing to reach squad selection times), there is no end point of success. The swimmers must undertake meter after meter of 'mindful swimming' in order to embody swimming habits that enhance fitness, efficiency and speed. This process of 'mindful swimming' resonates with the phenomenological focus on the mind-body linkage and also draws on the use of the kinaesthetic and proprioceptive senses to highlight how movement is experienced. This was encapsulated by Stephen (20) in describing how these athletes, who already have a high level of technical skill, must still mindfully be engaged in 'doing' swimming:

...for me personally, I'm very aware of what's going on in my body when I swim...very aware of where my body is. It's like, you can swim with your hips really low so l'll, when we did that set the other week I'm fully tense so that I'm keeping my hips high in alignment so they're not causing extra drag, like, that's my thought the whole time, just staying in a good strong position.

Stephen refers here to actively having to engage his core muscles to keep his hips high in the water during a challenging swimming set, to ensure he remains technically efficient and as fast as possible. In a follow-up session, Stephen elaborated on the active nature of 'doing': 
On a good day I will be engaged in every single stroke. I'll be thinking about where my hand goes in, what my catch is like, what position my elbow is in, where my hips are. I am always switched on and thinking about what I'm doing in this situation. There is always something to focus on in more detail. (Stephen, 20)

Similarly, several swimmers identified the need to stay mindfully involved in what they were doing, in order to keep stroke technique, efficiency and therefore speed at optimal levels. This active engagement resonates with a form of dys-appearance (Leder, 1990) or euappearance (Zeiler, 2010), as the active process of engaging with technique brings certain parts of the body directly into consciousness via intentionality. Elements of this could be discomforting, as Scott recalled, noting how adjusting his freestyle technique resulted in: 'using muscles that [he] hadn't been using before, and so [he] got really bad aches and stuff in [his] shoulders'. Scott's shoulders came to occupy the 'to' in Leder's (1990) 'from-to' structure, where attention was firmly directed towards feelings in his shoulders as opposed to outward toward the world, until his new technique became incorporated into his bodyschema and his shoulders receded into the background once again. Such sensory engagement results in positive sensory experiences of eu-appearance (Zeiler, 2010) where the body feels good, efficient and fast:

You just feel like, streamlined and there is almost like no drag on you, you just like, you can just pull and just, feels like you can just keep on going, like, I think the only way you can say it is just feeling smooth like. It just feels effortless, like swimming along is just like nothing holding you back (Charles, 20).

When everything is good you feel like you are on top of the water, if you know what I mean. When, when your technique is there you feel like you feel powerful, like you...feel like you're not pulling yourself through the water you're just like, over the water if you know what I mean (Wade, 20).

The social aspects of somatic learning should also be noted, for it was regularly observed how the swimmers would help each other, for example, in offering stroke technique advice, such as when Frank, a butterfly swimmer, was working with Eddie, an individual medley (IM) swimmer, on his butterfly technique. Similarly, the backstroke community (men and women) would get together to work on starts or turns (Fieldnotes, February 2018). In these situations, the swimmers would draw on other swimmers' differing styles and experiences to help engage in a process of technical improvement, as the following fieldnote reveals: 
The backstroke community then gathers to work on their starts. There is Stephen, Wade, Remy, Scott and Pepper all helping to analyse and coach each other. Nick is doing some filming as well to give each of the guys some visual feedback. This shared learning and coaching is great to see as they all genuinely enjoy helping each other and want to see each other improve (Fieldnote, $13^{\text {th }}$ February 2018 AM).

Through such interactions and the sharing of information between swimmers, as well as between coaches and swimmers, skills and techniques are developed and refined. The learning and development of these techniques is therefore intersubjective and intercorporeal, shaped by the various discourses, practices, and social relations that form the performance swimming lifeworld.

It should be emphasized, however, that despite attaining a high level of technical proficiency at certain times, such skills and level of performance are only temporary; there is no fixed end point at which corporeal skills are developed and sedimented once-and-for-all (Allen-Collinson et al., 2018). The fluid and evolving nature of 'doing' renders it difficult to ascertain a point at which technical efficiency is achieved. The goal for these highly skilled athletes thus becomes less about learning new skills or techniques and more about constantly refining their current skillset through repetitive, mindful action over the entire course of a swimmer's career as the following section highlights.

\section{The 'mindful' changing or development of technique}

Changing or developing technique is a difficult process, in which the swimmers must challenge their own proprioceptive memory and habits. Usually, the swimmers undertake one or two specific technique and skill development sessions per week, with technical work also built into warm-ups, preparatory work and/or swim-downs. Technical work is also incorporated into most sessions at the beginning of the season (first 3-4 weeks); a time regarded by the coaches as optimal for this kind of work, with the swimmers being freshly returned from a break. Changing technique involves a highly conscious and active process through which bodily memories are changed over time through the working and reworking of bodies (Spencer, 2009). The swimmers and coaches work together on this technical development, casting their gaze over others, scrutinizing their technique, and offering suggestions on how to increase the level of technical swimming performance. For example, a hand that over-reaches on a 
backstroke entry (to the water) may well feel like it is in the right place to the swimmer, whereas in reality it is actually behind the head rather than in line with the shoulder. To change this, the swimmers may utilize video recordings to identify the technical fault and give them the opportunity subsequently to deploy their embodied knowledge in a different way. As Crossley (2007: 89) argues: 'agents can learn to find parts of their body and mobilize them in new ways'. Technique correction therefore requires a state of watchfulness and mindfulness, where a swimmer's intentionality and action are directed to specific body parts and feelings. In correcting such over-reaching in backstroke, for instance, swimmers were often left with the sensation that their arm was entering the water at ninety degrees to their shoulder when actually they had moved it only a few centimeters from behind their head to in-line with the shoulder. This example highlights how the process of change often results in an alien or disjointed feeling, and bodily dys-appearance (Leder, 1990) also emphasized by Wade (20):

When you first do it, it makes you're stroke just feel weird, but then obviously if you keep doing it and keep doing it, you get used to it. But, the first initial change, it, it throws you a bit, like your like, 'ok my stroke feels a bit weird now' but then you get back used to it again.

This process of developing somatic ways of knowing (Allen-Collinson et al., 2016), involving change, refinement and evolution, is far from linear, and often results in repetitive steps backwards and forwards, as Peter (21) emphasized:

Doing technique is probably the most awkward thing that you can do in swimming, because when you swim for so long, you get into a routine of what 'normal' is. So, you get into the rhythm... and then all of a sudden someone tells you you're doing it wrong, or, 'change this' ... So, it completely shakes off your feel. It's very much a psychological game as well, because changing a thing technically is easy, but believing that what you're changing is actually beneficial is the big barrier that you need to overcome. Also, to have the psychological willpower to carry on, because technique doesn't change overnight, and adapting to a technical change typically takes you back one step. The hope is that you go two steps forward, but a lot of the problem is that when you go back that one step, people stop, and they go back to old ways because it doesn't feel right or...you feel disjointed... So, yeah, your body just feels awkward, and it's because of that awkwardness that you often revert back to your old technique.

Peter's quote again highlights the linkage between mind and body in the 'doing' of swimming. He emphasizes how, when making technical adjustments, the swimmers must be 'in-tune' 
with the movements of their body and must continue to focus on the new technique for an extended period of time in order for adjustments to their habit-body to be made. Somatic attunement (Allen-Collinson and Owton, 2015) and listening to their bodies thus become important embodied processes for these athletes as they negotiate changes in technique, but also different types of training, and periods of the season. This attunement results in a greater understanding of their embodied capabilities. Such understanding can, however, be 'lost' during times out of the water at the end of a season, or as a result of injury, where levels of physical conditioning can diminish and a 'feel for the water' is lost. These instances can engender in the swimmers a sense of having forgotten how to 'do' swimming, and of having lost their embodied swimming hexis, as Peter (21) indicated:

Swimming's one of those things that you lose, unfortunately. So, I have two days out of the water, and I lose all feel and catch. It's one of those sports that's, it's gone as quick as [pause]. So, if you're out a day, you will feel the consequences of being out a day, which is why we have such a vigorous schedule.

Furthermore, Peter's and the other swimmers' accounts drew attention to the habitual nature of 'doing' swimming. Although the swimmers often referred to technique as a habit, the fluid and evolving nature of 'doing' adds illustrative weight to Merleau-Ponty's (2002) concept of habit, not as a fixed, mechanical, response to stimuli, but as an 'agentic', flexible power of responding to situations. Habit therefore is a form of embodied and practical understanding, or know-how, that is grasped and incorporated into one's bodily schema, often as a tacit and practical principle of action (Crossley, 2001); a form of bodily knowledge resulting from physical sensations and increased bodily awareness (Lawrence, 2012). This flexibility of habit is highlighted by how in practice the swimmers engage with a variety of drills or periods of trial and error that are designed to give them the opportunity to direct their intentionality to a specific element of their technique or body part that needs scrutiny, so that over time this leads to the effective performance of refined skilled movement. As this new movement pattern becomes habituated, the swimmers no longer have to focus their intentionality on the new component of their technique; it has been incorporated into their body schema and as a result often recedes into background awareness, allowing them to shift their attention to other technical or tactical elements. This point was emphasized by Clint (20) who in reference to changing his technique indicated how this may initially make him slower as he had to think about the change but: 
...when it actually then starts to become inbuilt again, you don't actually have to think about it and you start going quicker because you can focus on the rest of the stuff that you're doing, [for example] the pace that you're [swimming] at.

This complex, fluid dynamic of 'doing' helps illuminate how skillful action is developed as a whole.

Furthermore, the spatial dimension is important in 'doing' swimming and changing technique, for as Moya (2014: 1) notes, with reference to Merleau-Ponty (2002), the lived human body relates to space that is also 'lived'. Habit thus presupposes a form of understanding that the body has in terms of operating within a certain world, establishing an operant or operative intentionality (Merleau-Ponty, 2002) which refers to the pre-reflective and corporeal link that a subject has with the world. The corporeal subject (swimmer) inhabits a world (aquatic) that provokes certain questions that need resolving. The body therefore must adapt in order to solve these questions. In the case of these swimmers that question most often relates to how to move through water faster. As Gallagher and Zahavi (2008: 138) note: 'the environment therefore calls forth a specific body-style so that the body works with the environment and is included in it. The posture the body adopts in a situation is its way of responding to the environment'. Similarly, Ingold (2000: 230) notes how our knowledge of the environment undergoes continuous formation in the very course of our moving in it. The body therefore must anticipate and react to a constantly changing environment, which is perhaps made even more pertinent by the fluidity and viscosity of water. Matthew (22) eloquently sums up this constant fluid, evolving, dynamic interaction between the swimming body-mind and environment:

I like the fact that we are in water and that there is nothing stable about water. It's always moving, and it's the same with how you feel and how you approach every day. You have to just constantly keep changing the way you approach things.

Habit therefore bears a direct relation to the dialogue between subject and environment. Competitive swimming thus involves embracing an embodied feel in addition to objective knowledge; one cannot become a competitive swimmer simply by reading about it, one must actively engage with the aquatic world in order to 'do' swimming. As Ingold (2000) points out, the skill of doing is grounded in the 'attentive perceptual involvement' with one's environment: 
...foundations of skill lie in the irreducible condition of the practitioner's embeddedness in an environment...[where] through repeated practical trials, and guided by observations [of accomplished practitioners], he [sic] gradually gets the 'feel' of things for himself - that is, he learns to fine-tune his own movements so as to achieve the rhythmic fluency of the accomplished practitioner (Ingold, 2000: 353).

Thus, swimming-body pedagogics highlight the indissoluble body-mind-world nexus lying at the heart of sociological phenomenology.

\section{Pre-reflexive, 'switched off' swimming}

Finally, it is important to note that the swimmers did also allude to times when they 'switched off', usually during recovery sessions, disengaging the mind from 'doing' and paying little attention to their body, in order to escape the pressures of competitive swimming and simply be present in the aquatic moment, as both Mary-Jane (22) and Jessica (20) reflected:

Mary-Jane: I think, well there is [sic] two things. Firstly, swimming training does put a lot of pressure on the body and mind, so it's difficult to stay $100 \%$ engaged for 12 sessions including gym a week. That's one reason why I don't think you can, but also because in a race pace session you train to get better. In a speed session there's that focus of front-end speed $^{2}$. In a recovery session, well sometimes the point of a recovery session is just to not think. So, entering that, if you know it's a recovery session, you're not going to be as focused...

Like a recovery session, it's ok to switch off. It's ok to not concentrate, or to not care, or to have loads of rest, or talk, you know what I mean?

Jessica: I kind of think they are written [by the coaches] to not really think about as well. So, say if you are going for a run, you go for a hard sprint...or you choose to go for a nice stroll around a park...you're not really thinking when you are strolling but when you are running at a pace you're really focused on getting better and fitter. Whereas when you're walking you almost feel a pleasure.

This idea of 'letting go' and 'just swimming' resonates with Leder's (1990) notion of the 'dis-appearing' mode of being-in-the-world. The swimmer's attention is drawn away from their own body and out towards the world. As a result, bodily awareness recedes into the background, evading explicit exploration. Such corporeal 'absence' in which the swimmers'

\footnotetext{
${ }^{2}$ Front-end Speed refers to the pace that the swimmers would target during the first part of their races. For example, it might be the pace they would like to swim for the $1^{\text {st }} 50 \mathrm{~m}$ or $1^{\text {st }} 100 \mathrm{~m}$ of a $200 \mathrm{~m}$ event.
} 
focus is from the body to the objects of experience, allows the swimmers to enjoy 'just being' in their aquatic lifeworld. Such a position is still, however, only possible when mind and body act harmoniously with the environment or situation in which people are engaged. Such a position further highlights the mind-body-world nexus, as well as the importance of the 'incorporation' of skills and habits into one's body schema, as a form of body pedagogics, so that the sensory bodily actions of swimming become part of the swimmers own highly technical pre-reflexive bodily know-how.

\section{Conclusion}

The purpose of this paper has been to investigate how the embodied doing of swimming and a swimmer's ability to inhabit the competitive swimming lifeworld are dependent on the acquisition of a specific set of swimming techniques and skilled behaviors, acquired through a complex, cyclical process. This demands experimentation, discovery and the ability constantly to adjust and adapt, depending on the practice (Downey, 2005), as well as linking mind, body and world. Skill acquisition and 'doing' therefore is not just about 'prestigious imitation' as Mauss (1979) described, but also about the social nature of such somatic learning, as requiring input from others (Shilling, 2007; Throsby, 2016). In this particular case, coaches and other swimmers were involved, as illustrated above by, for example, the group of specialist backstroke swimmers, whose members worked together in order to enhance each other's learning and technical development. In this way the body is socialized into physical-culturally specific ways of being, in that individuals incorporate patterns of action into their 'flesh', and that flesh is conditioned by the activity in an interlinked process.

The findings described in this article derive from a study that inevitably has some limitations, being a relatively small-scale project on senior competitive swimmers (aged 1822) with a primarily white-British background (with the exception of four athletes two of whom were not interviewed, and two white-Irish athletes) from one performance swimming programme. Whilst the aim of the sociological-phenomenological project was never generalization to wider populations, the findings do address qualitative-appropriate conceptualizations of 'naturalistic' and 'analytic' generalizability and transferability (Smith, 2018) to other physical-cultural domains. The data thus resonate with other research studies that focus upon the acquisition and habituation of a specific set of body techniques that afford sportspeople the opportunity to participate in, as well as shape their various sporting bodies 
into corporeally proficient bodies, for example, in the Brazilian Jiu-Jitsu grappler's body (Hogeveen, 2013), the circuit training body (Crossley, 2004), or the marathon swimming body (Throsby, 2016).

One of the most salient findings from the project was the extent to which the 'doing' and 'learning how' of swimming are so strongly socially, and physical-culturally shaped. This offers insight into the dynamic and dialectical nature of embodied action as well as the centrality and malleability of our embodied being-in-the-world. Our embodiment thus offers us a unique reference point from which to experience the world, as well as simultaneously being shaped on an ongoing basis by time, space and our relation to others. Merleau-Ponty's (2002) phenomenology is a powerful resource in analyzing this dynamic, and acknowledging not only the centrality of the body to our experiencing of the world, but also the capacity for humans to expand their body schemas through the learning of new skills, or the development and refinement of extant skills. From this standpoint one's own body, or le corps propre, anchors the swimmers in, and allows them to engage with the world around them. In emphasizing the centrality of the body in lived experience, Merleau-Ponty (1968), in his later work, recast the very notion of being-in-the-world as flesh-of-the-world, or 'chair', better to convey what Author 2 and another (2014: 549) term our 'corpo-reality'. It is this 'corporeality' that allows the swimmers to engage with the different practices of their aquatic environment in order to bring about, through intelligent practice (Marchand, 2010), both reflective and pre-reflective skilled 'doing'. As a result, the swimmers develop a tacit, embodied knowledge or know-how that forms their aquatic corporeal schema. The swimming body is thus a lived body that is conscious, active, reflexive, and fundamentally linked to the world as part of a single mind-body-world system. It should be cautioned, however, that skill and habit are not permanent, or learnt once and for all, and as well as offering the capacity to being developed and refined, they can also degenerate, as Peter noted when describing how the swimming 'feel' can be lost after only two days out of the aquatic environment. This highlights how the competitive swimming lifeworld is a dynamic, fluid, lived-through structure in-process, that constantly '(de-)evolves' as an effect of the interactions, or lack of interactions that an agent has with others, and with their physical aquatic environment.

Responding to calls to explore further the domain of 'body pedagogics' (Shilling, 2017), and drawing on original findings, this article offers a novel theoretical approach to the sociologically under-researched physical-cultural domain of competitive swimming, with a 
particular focus on the everyday training routines involved. Here, we have investigated how swimmers develop some of the practical, embodied ways of knowing how to 'do' swimming, developed and refined by competitive swimmers, as 'sensate, suffering, skilled, sedimented, and situated creature[s] of flesh and blood' (Wacquant 2015: 1). The study contributes to a small, but growing corpus that employs a sociological-phenomenological approach to subject to detailed scrutiny the everyday and often taken-for-granted aspects of sporting embodiment. It further develops a corporeally and sensorially grounded 'carnal sociology' (Crossley, 1995) that draws on the existential phenomenology of Merleau-Ponty $(1968,2002)$. Beyond the academic contribution, the research findings can provide competitive-swimming coaches with insights garnered from an in-depth study of the lifeworlds of the athletes whom they seek to train and develop, for example, on the importance of the mind-body nexus in somatic learning, the role of mindfulness, and also the need to accord athletes the time and space to engage in 'switched-off' swimming.

\section{Notes}

1. Eu-appearance. Zeiler (2010) uses the Greek prefix $e u$ to highlight the experience of the body as well, easy or good.

2. Front-end speed refers to the pace that the swimmers would target during the first part of their races. For example, it might be the pace they would like to swim for the first $50 \mathrm{~m}$ of a $100 \mathrm{~m}$ event, or the first $100 \mathrm{~m}$ of a $200 \mathrm{~m}$ event.

\section{References}

Allen-Collinson J (2009) Sporting embodiment: Sports studies and the (continuing) promise of phenomenology. Qualitative Research in Sport and Exercise 1(3): 279-296.

Allen-Collinson J (2011) Feminist phenomenology and the woman in the running body. Sport, Ethics and Philosophy 5(3): 297-313.

Allen-Collinson J (2016) Breathing in Life. Phenomenological perspectives on sport and exercise. In: Sparkes AC and Smith B (eds) Routledge Handbook of Qualitative Research Methods in Sport and Exercise. London: Routledge, 11-23.

Allen-Collinson J and Owton H (2015) Intense embodiment: Senses of heat in women's running and boxing. Body \& Society 21(2): 245-268.

Allen-Collinson, J, Owton, H and Crust, L (2016) Opening up dialogues and airways: Using vignettes to enrich asthma understandings in sport and exercise. Qualitative Research in Sport, Exercise \& Health 8(4): 352-364. 
Allen-Collinson J, Crust L and Swann C (2018) 'Endurance work': Embodiment and the mindbody nexus in the physical culture of high-altitude mountaineering. Sociology 52(6): 1324-1341

Bloor M (2001) Techniques of validation in qualitative research: A critial commentary. In: Emerson RM (ed.) Contemporary field research. Prospect Heights, IL: Waveland Press, pp. 383-396.

Braun V and Clarke V (2013) Successful Qualitative Research: A practical guide for beginners. London: Sage.

Braun V and Clarke V (2019) Reflecting on reflexive thematic analysis. Qualitative Research in Sport, Exercise and Health. doi:10.1080/2159676X.2019.1628806.

Brentano F (1874/1995) Psychology from an Empirical Standpoint. London: Routledge.

Crossley N (1995) Merleau-Ponty, the elusive body and carnal sociology. Body \& Society 1(1): 43-63.

Crossley N (2001) The Social Body: Habit, identity and desire. London: Sage.

Crossley N (2004) The Circuit Trainer's Habitus: Reflexive Body Techniques and the Sociality of the Workout. Body \& society 10(1): 37-70.

Crossley N (2007) Researching embodiment by way of 'body techniques'. The Sociological Review 55(s1): 80-94.

Dale GA (1996) Existential phenomenology: Emphasizing the experience of the athlete in sport psychology research. The Sport Psychologist 10(4): 307-321.

Downey G (2005) Learning Capoeira: Lessons in cunning from an Afro-Brazilian art. Oxford: Oxford University Press.

Evers C (2006) How to surf. Journal of Sport and Social Issues 30(3): 229-243.

Fisher L and Embree L (2000) Feminist Phenomenology. Dordrecht: Kluwer Academic Publishers.

Gallagher S and Zahavi D (2008) The Phenomenological Mind. London: Routledge.

Hockey J and Allen-Collinson J (2016) Digging In. The sociological phenomenology of "doing endurance" in distance running. In: Bridel W, Markula P and Denison J (eds) Endurance Running. A socio-cultural examination. London: Routledge, pp. 227-242. 
Hogeveen B (2013) 'It's about your body recognising the move and automatically doing it': Merleau-Ponty, habit and Brazilian Jiu-Jitsu. In: Garcia RS and Spencer DC (eds) Fighting Scholars: Habitus and Ethnographies of Martial Arts and Combat Sports. London: Anthem Press, pp. 79-94.

Husserl E (1970) The Crisis of European Sciences and Transcendental Phenomenology, Evanston, IL: Northwestern University Press.

Ingold T (2000) Perception of the Environment: Essays in Livelihood, Dwelling and Skill. London: Routledge.

Kerry DS and Armour KM (2000) Sport sciences and the promise of phenomenology: Philosophy, method, and insight. Quest 52(1): 1-17.

Kvale S and Brinkmann S (2009) Interviews: Learning the Craft of Qualitative Research. Thousand Oaks, CA: Sage.

Lang M (2010) Surveillance and conformity in competitive youth swimming. Sport, Education and Society 15(1): 19-37.

Lawrence RL (2012) Intuitive knowing and embodied consciousness. New Directions for Adult and Continuing Educations 2012(134): 5-13.

Leder D (1990) The Absent Body. Chicago, IL: University of Chicago Press.

Marchand THJ (2010) Making knowledge: explorations of the indissoluble relation between minds, bodies and environments. Journal of the Royal Anthropological Institute 21(1): $2-20$.

Mauss M (1979) Sociology and Psychology. London: Routledge \& Kegan Paul.

McMahon J and Penney D (2013a) Body pedagogies, coaching and culture: three Australian swimmers' lived experiences. Physical Education and Sport Pedagogy 18(3): 317-335.

McMahon J and Penney D (2013b) (Self-) surveillance and (self-) regulation: Living by fat numbers within and beyond a sporting culture. Qualitative Research in Sport, Exercise and Health 5(2): 157-178.

McMahon J, Penney D and Dinan-Thompson M (2012) 'Body practices-exposure and effect of a sporting culture?' Stories from three Australian swimmers. Sport, Education and Society 17(2): 181-206. 
McNarry G, Allen-Collinson J and Evans A (2019) Reflexivity and bracketing in sociological phenomenological research: Researching the competitive swimming lifeworld. Qualitative Research in Sport, Exercise and Health 11(1): 138-151

Merleau-Ponty M (1968) The Visible and the Invisible. Evanston: Northwestern University Press.

Merleau-Ponty M (2002) Phenomenology of Perception. London: Routledge.

Moya P (2014) Habit and embodiment in Merleau-Ponty. Frontiers in Human Neuroscience 8: 1-3. doi:10.3389/fnhum.2014.00542

Nesti MS (2004) Existential Psychology and Sport. London: Routledge.

Purser A (2019) Dancing intercorporeality: A health humanities perspective on dance as a healing art. Journal of Medical Humanities 40(2): 253-263.

Ravn S and Christensen MK (2014) Listening to the body? How phenomenological insights can be used to explore a golfer's experience of the physicality of her body. Qualitative Research in Sport, Exercise and Health 6(4): 462-477.

Schütz A (1967) Phenomenology of the Social World. Evanston, IL: Northwestern University Press.

Shilling C (2007) Sociology and the body: Classical traditions and new agendas. The Sociological Review 55(s1): 1-18.

Shilling C (2010) Exploring the society-body-school nexus: Theoretical and methodology issues in the study of body pedagogics. Sport, Education and Society 15(2): 151-167.

Shilling C (2012) The Body and Social Theory: Theory, culture and society. London: Sage.

Shilling C (2017) Body pedagogics: Embodiment, cognition and cultural transmission. Sociology 51(6): 1205-1221.

Smith B (2018) Generalizability in qualitative research: Misunderstandings, opportunities and recommendations for the sport and exercise sciences. Qualitative Research in Sport, Exercise and Health 10(1): 137-149.

Sokolowski R (2000) Introduction to Phenomenology. Cambridge: Cambridge University Press.

Spencer DC (2009) Habit(us), body techniques and callusing: An ethnography of Mixed Martial Arts. Body \& society 15(4): 119-143. 
Throsby K (2016) Immersion: Marathon Swimming, Embodiment and Identity. Manchester: Manchester University Press.

Tracy SJ (2010) Qualitative quality: Eight 'big-tent' criteria for excellent qualitative research. Qualitative Inquiry 16(10): 837-851.

Vaittinen A (2014) Varieteis of Embodied Knowing: An ethnographic study of Mixed Martial Arts. (Unpublished doctoral dissertation) Newcastle, UK: Newcastle University.

Wacquant L (2015) For a sociology of flesh and blood. Qualitative Sociology 38(1): 1-11.

Wagner HR (1973) The scope of phenomenological sociology: Considerations and suggestions. In: Psathas G (ed.) Phenomenological Sociology: Issues and applications. London: John Wiley, pp. 61-87.

Wheaton B (2002) Babes on the beach, women in the surf: Researching gender, power and difference in the windsurfing culture. In: Sugden J and Tomlinson A (eds) Power games: A Critical sociology of sport. London: Routledge, pp. 240-266.

Zeiler K (2010) A phenomenological analysis of bodily self-awareness in the experience of pain and pleasure: on dys-appearance and eu-appearance. Medicine, Health Care and Philosophy 13(4): 333-342. 